\title{
Dysregulation of miR-200s clusters as potential prognostic biomarkers in acute myeloid leukemia
}

Jing-dong Zhou ${ }^{1,2 \dagger}$, Liu-chao Zhang ${ }^{3 \dagger}$, Ting-juan Zhang ${ }^{1,2}$, Yu Gu ${ }^{1,2}$, De-hong Wu ${ }^{4}$, Wei Zhang ${ }^{1,2}$, Ji-chun Ma ${ }^{2,5}$, Xiang-mei Wen ${ }^{2,5}$, Hong Guo ${ }^{2,5}$, Jiang Lin ${ }^{2,5^{*}}$ and Jun Qian ${ }^{1,2^{*}}$

\begin{abstract}
Background: Increasing studies showed that miR-200 family (miR-200s) clusters are aberrantly expressed in multiple human cancers, and miR-200s clusters function as tumor suppressor genes by affecting cell proliferation, self-renewal, differentiation, division and apoptosis. Herein, we aimed to investigate the expression and clinical implication of miR200s clusters in acute myeloid leukemia (AML).
\end{abstract}

Methods: RT-qPCR was performed to detect expression of miR-200s clusters in 19 healthy donors, 98 newly diagnosed AML patients, and 35 AML patients achieved complete remission (CR).

Results: Expression of miR-200a/200b/429 cluster but not miR-200c/141 cluster was decreased in newly diagnosed AML patients as compared to healthy donors and AML patients achieved CR. Although no significant differences were observed between miR-200s clusters and most of the features, low expression of miR-200s clusters seems to be associated with higher white blood cells especially for miR-200a/200b. Of the five members of miR-200s clusters, low expression of miR-200b/429/200c was found to be associated with lower CR rate. Logistic regression analysis further revealed that low expression of miR-429 acted as an independent risk factor for CR in AML. Based on Kaplan-Meier analysis, low expression of miR-200b/429/200c was associated with shorter OS, whereas miR-200a/141 had a trend. Moreover, multivariate analysis of Cox regression models confirmed the independently prognostic value of miR-200b expression for OS in AML.

Conclusions: Expression of miR-200a/200b/429 cluster was frequently down-regulated in AML, and low expression of miR-429 as an independent risk factor for CR, whereas low expression of miR-200b as an independent prognostic biomarker for OS.

Keywords: miR-200, Expression, Prognosis, Acute myeloid leukemia

\footnotetext{
*Correspondence: linjiangmail@sina.com; qianjun0007@hotmail.com

†ing-dong Zhou and Liu-chao Zhang contributed equally to this work

1 Department of Hematology, Affiliated People's Hospital of Jiangsu University, 8 Dianli Rd., Zhenjiang 212002, Jiangsu, People's Republic of China ${ }^{5}$ Laboratory Center, Affiliated People's Hospital of Jiangsu University, 8 Dianli Rd., Zhenjiang 212002, Jiangsu, People's Republic of China

Full list of author information is available at the end of the article
} 


\section{Background}

Acute myeloid leukemia (AML) is a highly heterogeneous malignant hematological disorder with complex molecular pathophysiology. Although the treatment strategies against AML have been updated in the past decades, the majority of patients eventually succumb to relapse after induction chemotherapy [1]. Clinical outcome of AML remains unsatisfactory especially in those with specific karyotypes/biomarkers such as inv(3)(q21q26.2), t(6;9) (p23; q34), 11q abnormalities other than $\mathrm{t}(9 ; 11),-5 /$ del(5q), -7, TP53 mutations, FLT3-ITD mutations, C-KIT mutations, WT1 overexpression, and $B A A L C$ overexpression [2-4]. The development of effective therapeutic options against AML relies on mechanistic understanding of AML biology, especially in molecular regulators of AML pathogenesis and molecular predictor of AML prognosis [5].

MicroRNAs, a class of small (19-22 nucleotides) single-stranded RNAs, negatively regulate various genes by targeting $3^{\prime}$-untranslated region ( $3^{\prime}$-UTR) of mRNAs, thereby facilitating translational silencing or degradation of targeted genes [6]. Mounting evidences have implicated that microRNAs play crucial roles in regulating many fundamental and biological processes including cancer development [7]. Moreover, microRNAs have been reported as novel biomarkers for diagnosis and prognosis, and regarded as potential therapeutic targets in AML [8]. For instance, recent studies implicated that several microRNAs such as $m i R-216 b, m i R-362-5 p$, $m i R-217$, and $m i R-193 b$ were prognosis-related predictors in $A M L$ and may involve in AML biology [9-12].

The miR-200 family (miR-200s) clusters includes five members (miR-200a, miR-200b, miR-200c, miR-141, and $m i R-429)$ and can be divided into two clusters ( $m i R-200 a / b / 429$ cluster and $m i R-200 c / 141$ cluster) based on chromosomal location (chromosome 1p36 and chromosome 12p13) [13]. Numerous studies showed that $m i R-200 \mathrm{~s}$ clusters are aberrantly expressed in multiple human cancers, and $m i R-200 s$ clusters function as tumor suppressor genes by affecting cell proliferation, self-renewal, differentiation, division and apoptosis [14]. Although the tumor-suppressive roles of $m i R-200 s$ clusters have also been reported in solid tumors with prognostic value $[14,15]$, the expression and clinical implication of $m i R-200 s$ clusters in AML remains poorly revealed.

In this study, we investigated expression of $m i R-200 \mathrm{~s}$ clusters in AML patients except for acute promyelocytic leukemia (APL), and found that low expression of $m i R$ $200 s$ clusters acted as potential prognostic biomarkers in AML.

\section{Methods}

Patients and treatment

A total of 98 de novo AML patients except for APL and 19 healthy donors were enrolled in this study. Bone marrow (BM) was collected from all the patients at diagnosis time as well as 35 patients at complete remission (CR) time. AML was diagnosed based on the FrenchAmerican-British (FAB) and 2016 revised World Health Organization (WHO) criteria [16, 17]. All the patients received chemotherapy as reported [18]. Induction chemotherapy therapy was 1-2 courses of daunorubicin combined with cytarabine. Subsequent consolidation treatment after CR for younger patients included highdose cytarabine, mitoxantrone with cytarabine, and homoharringtonine combined with cytarabine, whereas for older patients received in an individualized manner decided by the physicians, such as CHG protocol (cytarabine, homoharringtonine, and G-CSF). This study was approved by the Ethics Committee of the Affiliated People's Hospital of Jiangsu University, and written informed consents were informed and signed by all participants in accordance with the Declaration of Helsinki Principles.

\section{Cytogenetic analysis and mutation detection}

BM cells were harvested after 1-3 days of unstimulated culture in RPMI 1640 medium (BOSTER, Wuhan, China) containing 20\% fetal calf serum (ExCell Bio, Shanghai, China). Cytogenetics for AML patients were analyzed at the newly diagnosis time by conventional R-banding method and karyotype risk was classified according to reported previously $[19,20]$. Hotspot mutations in NPM1, C-KIT, DNMT3A, N/K-RAS, IDH1/2, U2AF1, $S R S F 2$ and SETBP1 were detected by high-resolution melting analysis [21-25], whereas mutations in FLT3ITD and CEBPA were examined by DNA sequencing [26].

\section{RNA isolation and reverse transcription}

$\mathrm{BM}$ mononuclear cells (BMMNCs) were extracted as reported using Lymphocyte Separation Medium (Absin, Shanghai, China) [27]. According to the manufacturer's protocols, RNA was extracted from BMMNCs using the mirVana miRNA isolation kit (Ambion, Austin, TX, USA), and was synthesized to cDNA by reverse transcription using MiScript Reverse Transcription Kit (Qiagen, Duesseldorf, Germany).

\section{Real-time quantitative PCR}

The level of $m i R-200 s$ clusters was detected by real-time quantitative PCR (RT-qPCR) using miScript SYBR green PCR kit (Qiagen, Duesseldorf, Germany). The primers were $m i R-200 s$ specific (Additional file 1: Table S1) and the manufacturer-provided miScript universal primer 
(Qiagen, Duesseldorf, Germany). The programs for RTqPCR reactions were performed as reported [28]. U6 small nuclear RNA was selected as the endogenous normalizer detected by RT-qPCR using $2 \times$ SYBR Green PCR Mix (Multisciences, Hangzhou, China). Relative $m i R-200 s$ level was calculated by $2^{-\Delta \Delta C T}$ method. The healthy donors that possessed the minimal $\triangle C T$ between miR-200s (each member) and $U 6$ expression was selected as control, and was defined as $100 \%$ expression.

\section{Statistical analysis}

Mann-Whitney's U test was carried to compare the difference of continuous variables between two groups, whereas Pearson Chi square analysis/Fisher exact test were applied to compare the difference of categorical variables between two groups. The impact of miR200 s clusters expression on overall survival (OS) was analyzed by Kaplan-Meier analysis, and Cox regression models (univariate and multivariate analyses) were further used to determine the independently prognostic value of $m i R-200 \mathrm{~s}$ cluster expression. The effect of miR-200s clusters expression on CR was determined by Logistic regression analysis (univariate and multivariate analyses). All tests were two sided, and $P<0.05$ was defined as statistically significant. SPSS software 20.0 and GraphPad Prism 5.0 was used to conduct the statistical analyses in this study.

\section{Results}

\section{Expression of miR-200s in AML}

We analyzed miR-200s clusters expression in BM from 19 healthy donors, 98 AML patients, and 35 AML patients achieved CR by RT-qPCR. As presented in Fig. 1, expression of $m i R-200 a / 200 b / 429$ clusters but not $m i R-200 c / 141$ clusters was significantly decreased in AML patients as compared to healthy donors and AML patients achieved CR.

\section{Relationship between miR-200s and clinical features in AML}

To investigate clinical implication of miR-200s clusters expression, the whole-cohort patients were classified into two groups (high and low miR-200s clusters expression) based on the median level of each member of $m i R$ 200s clusters, respectively. We analyzed the association between each member of $m i R-200 s$ clusters expression and clinic-pathologic features including gender, age,
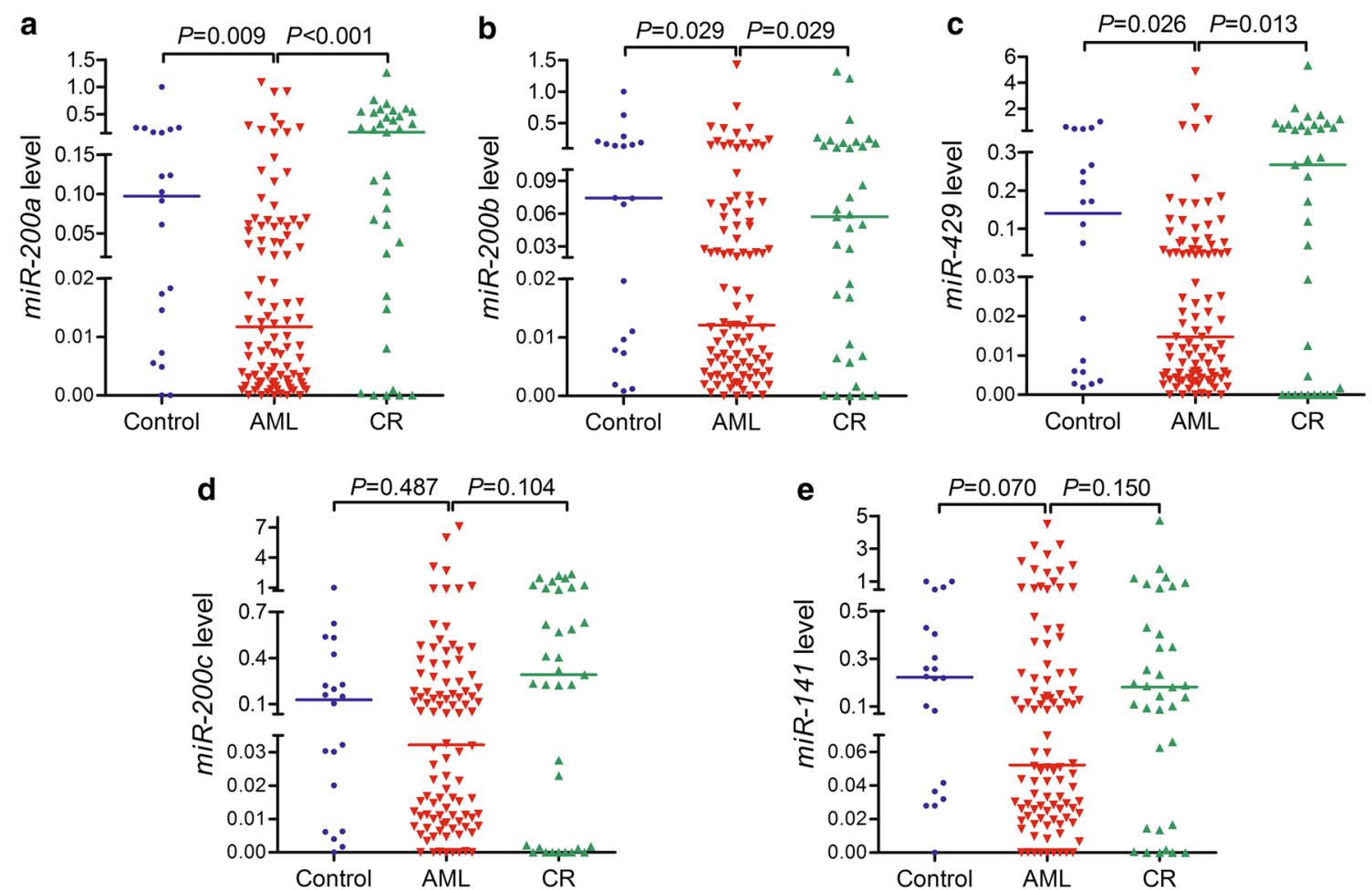

Fig. 1 Expression of miR-200s clusters in controls, newly diagnosed AML patients and AML patients achieved CR. a For miR-200a; b For miR-200b; c For miR-429; d For miR-200c; e For miR-141. The distributions of the miR-200s clusters expression in controls, newly diagnosed AML patients and AML patients achieved CR were presented with scatter plots. The median level of miR-200s clusters expression in each group was shown with horizontal line 


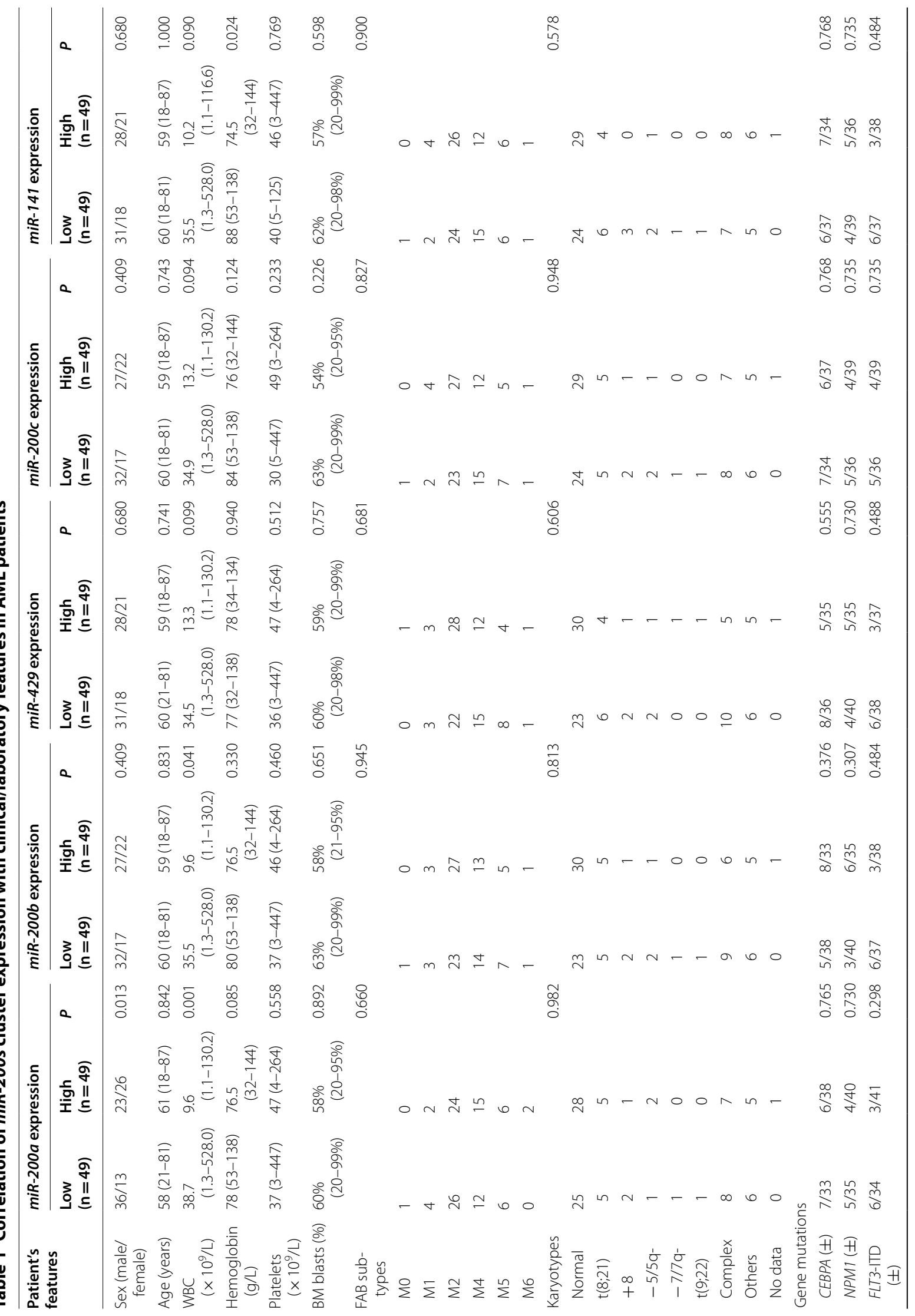




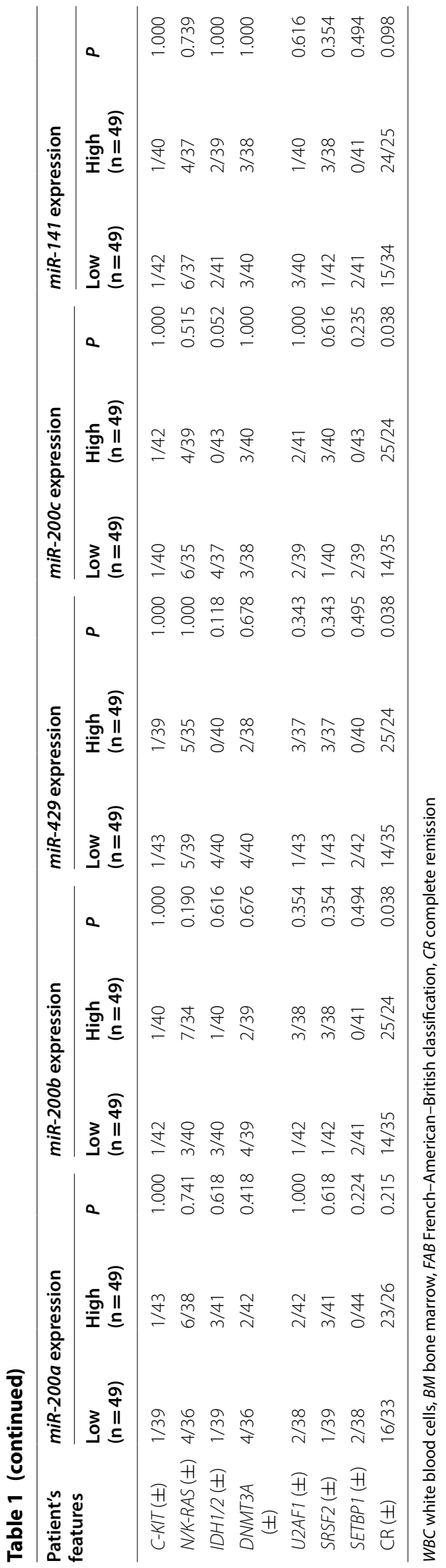


Table 2 Univariate and multivariate analyses of variables for overall survival in AML patients

\begin{tabular}{|c|c|c|c|c|c|c|c|c|}
\hline \multirow[t]{3}{*}{ Variables } & \multicolumn{4}{|l|}{ Complete remission } & \multicolumn{4}{|l|}{ Overall survival } \\
\hline & \multicolumn{2}{|l|}{ Univariate analysis } & \multicolumn{2}{|l|}{ Multivariate analysis } & \multicolumn{2}{|l|}{ Univariate analysis } & \multicolumn{2}{|l|}{ Multivariate analysis } \\
\hline & OR $(95 \% \mathrm{Cl})$ & $P$ & OR $(95 \% \mathrm{Cl})$ & $P$ & $\mathrm{HR}(95 \% \mathrm{Cl})$ & $P$ & $\mathrm{HR}(95 \% \mathrm{Cl})$ & $P$ \\
\hline miR-200a & $0.548(0.242-1.244)$ & 0.150 & $1.029(0.285-3.722)$ & 0.965 & $0.662(0.415-1.022)$ & 0.082 & $1.425(0.651-3.120)$ & 0.376 \\
\hline miR-200b & $0.384(0.167-0.885)$ & 0.025 & $0.823(0.199-3.401)$ & 0.788 & $0.511(0.319-0.819)$ & 0.005 & $0.524(0.305-0.902)$ & 0.020 \\
\hline miR-429 & $0.384(0.167-0.885)$ & 0.025 & $0.331(0.128-0.858)$ & 0.023 & $0.558(0.350-0.891)$ & 0.015 & $0.820(0.325-2.073)$ & 0.675 \\
\hline miR-200c & $0.384(0.167-0.885)$ & 0.025 & $0.977(0.149-6.400)$ & 0.981 & $0.606(0.380-0.965)$ & 0.035 & $0.649(0.190-2.217)$ & 0.491 \\
\hline $\operatorname{miR}-141$ & $0.460(0.201-1.050)$ & 0.065 & $0.594(0.192-1.833)$ & 0.364 & $0.695(0.437-1.104)$ & 0.123 & $1.152(0.582-2.279)$ & 0.684 \\
\hline Age & $4.229(1.742-10.266)$ & 0.001 & 4.555 (1.715-12.095) & 0.002 & $2.046(1.282-3.266)$ & 0.003 & $1.732(1.033-2.902)$ & 0.037 \\
\hline WBC & $2.367(1.015-5.520)$ & 0.046 & $1.846(0.715-4.767)$ & 0.206 & $2.002(1.253-3.199)$ & 0.004 & $1.560(0.925-2.629)$ & 0.095 \\
\hline Karyotype & $3.108(1.338-7.220)$ & 0.008 & $2.862(1.164-7.042)$ & 0.022 & 1.875 (1.295-2.715) & 0.001 & $1.874(1.210-2.902)$ & 0.005 \\
\hline CEBPA mutations & $0.526(0.160-1.731)$ & 0.290 & & & $0.870(0.413-1.829)$ & 0.713 & & \\
\hline NPM1 mutations & $0.833(0.207-3.358)$ & 0.798 & & & $1.200(0.516-2.793)$ & 0.672 & & \\
\hline FLT3-ITD mutations & $0.833(0.207-3.358)$ & 0.798 & & & $0.935(0.403-2.170)$ & 0.876 & & \\
\hline C-KIT mutations & $0.673(0.041-11.150)$ & 0.783 & & & $0.479(0.066-3.458)$ & 0.465 & & \\
\hline N/K-RAS mutations & $3.048(0.605-15.343)$ & 0.177 & & & $1.311(0.621-2.770)$ & 0.478 & & \\
\hline IDH1/2 mutations & Undetermined & 0.999 & & & $4.671(1.637-13.326)$ & 0.004 & $6.662(1.757-25.268)$ & 0.005 \\
\hline DNMT3A mutations & $1.391(0.240-8.057)$ & 0.712 & & & $1.590(0.634-3.987)$ & 0.323 & & \\
\hline U2AF1 mutations & Undetermined & 0.999 & & & $2.791(0.987-7.890)$ & 0.053 & $5.130(1.714-15.355)$ & 0.003 \\
\hline SRSF2 mutations & Undetermined & 0.999 & & & $1.934(0.693-5.400)$ & 0.208 & & \\
\hline SETBP1 mutations & $0.673(0.041-11.150)$ & 0.783 & & & $0.637(0.088-4.613)$ & 0.656 & & \\
\hline
\end{tabular}

OR odd ratio, $H R$ hazard ratio, $C l$ confidence interval. Variables including miR-200s cluster expression (Low vs. High), age ( $\leq 60$ vs. $>60$ years), WBC ( $\geq 30 \times 10^{9}$ vs. $<30 \times 10^{9} / \mathrm{L}$ ), karyotype (favorable vs. intermediate vs. poor), and gene mutations (mutant vs. wild-type). Multivariate analysis includes variables with $P<0.200$ in univariate analysis

white blood cell (WBC) counts, hemoglobin content, platelet counts, blasts (\%), FAB subtypes, karyotypes, and common gene mutations. As shown in Table 1, no significant differences were observed between miR-200s clusters expression and most of the features. However, low expression of $m i R-200$ s clusters seems to be associated with higher WBC counts especially for miR-200a/200b $(P=0.001$ and 0.041 , respectively). In addition, low expression of $m i R-200 a$ was related to male, whereas low expression of miR-141 was correlated with higher hemoglobin content $(P=0.013$ and 0.024 , respectively).

\section{Prognostic value of miR-200s in AML}

To observe the impact of miR-200s clusters expression on clinical outcome in AML, we first determined the association of each member of miR-200s clusters expression with CR. Of the five members of $m i R-200$ s clusters, low expression of $m i R-200 b / 429 / 200 c$ was found to be associated with lower $\mathrm{CR}$ rate (Table 1 , all $P=0.038$ ). Additionally, Logistic regression analysis was further performed to confirm and verify the effect of miR-200s clusters' expression on CR, and revealed low expression of $m i R-429$ as an independent risk factor for CR in AML (Table 2, $P=0.023$ ).
We next evaluated the correlation of each member of $m i R-200 s$ clusters expression with survival. Based on Kaplan-Meier analysis, low expression of miR$200 b / 429 / 200 c$ was associated with shorter OS, whereas $m i R-200 a / 141$ had a trend (Fig. 2). In addition, we also analyzed the impact of composite members of miR-200s clusters expression on OS by Kaplan-Meier analysis as shown in Fig. 3.

Since miR-200s clusters expression was associated with well-established prognostic factor such as WBC counts, we further conducted a Cox regression model adjusting for prognosis-related factors (age, WBC counts, karyotypic classifications, and gene mutations) for OS. Results showed that low expression of $m i R-200 b$ acted as an independent prognostic biomarker for OS $(P=0.020$, Table 2).

\section{Discussion}

In the current study, we for the first time investigated expression of $m i R-200 s$ clusters in AML, and revealed that most of the members of miR-200s clusters were down-regulated in de novo AML patients. Recently, $\mathrm{Li}$ et al. revealed that introduction of a pre-miR-200c reduced the expression of ZEB2 protein and inhibited the proliferation of human leukemia cell lines (HL-60, 

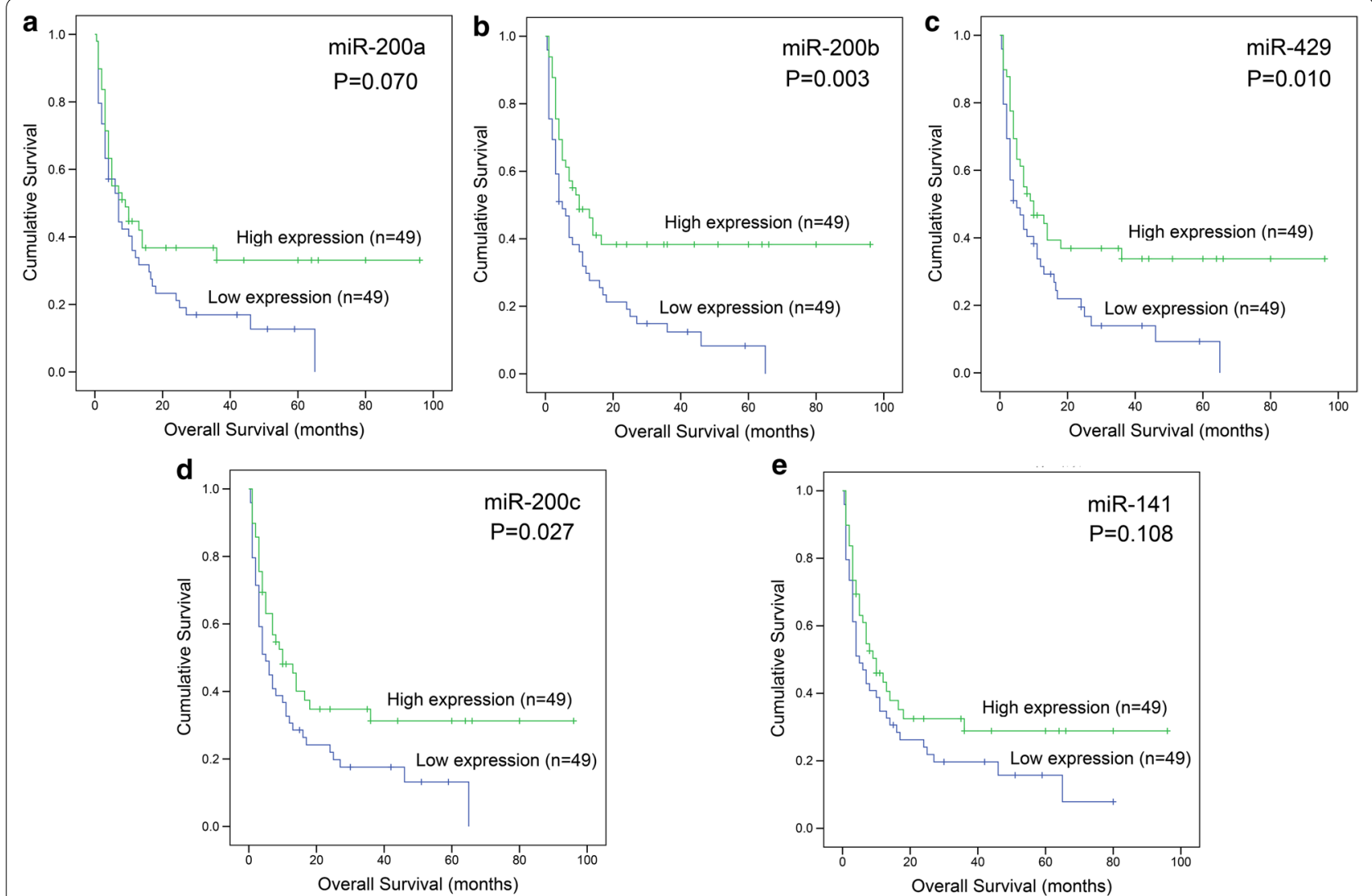

Fig. 2 Prognostic value of each member of miR-200s clusters expression in AML. a For miR-200a. b For miR-200b. c For miR-429. d For miR-200c. e For miR-141. Overall survival analyzed between two groups based on median level of each member of miR-200s clusters, and performed by KaplanMeier methods

MOLM-13, and THP-1), and mouse miR-200c significantly impaired the proliferation of mouse leukemia cells [29]. Taken together, these results emphasized the crucial role of miR-200s clusters in leukemogenesis. Although the biological role of $m i R-200 \mathrm{~s}$ clusters in AML was less studied, tumor suppressor roles of miR-200s clusters have been identified in a variety of human solid cancers, such as bladder cancer, gastric cancer, colorectal cancer, breast cancer, ovarian cancer, endometrial cancer, pancreatic cancer, gliomas, hepatocellular carcinoma, and lung cancer $[14,30]$. The $m i R-200$ s clusters were reported as key inhibitors of epithelial-to-mesenchymal transition by directly targeting transcriptional repressors of E-cadherin, ZEB1, and ZEB2 [13]. Moreover, miR-200s clusters also played crucial roles in the repression of cancer stem cells self-renewal and differentiation, modulation of cell division and apoptosis, and reversal of chemoresistance $[14,30]$. Notably, in some other hematological malignancies, expression or biological role of miR-200s clusters has been preliminary studied. For instance, Choi et al. reported that miR-200c was decreased in patients with myelodysplastic syndrome (MDS) [31]. González-Gugel et al. revealed that down-regulation of mmu-miR-30a and mmu-miR-141 as well as hsa-miR-193b clearly contributed to enhance the expression of Smoothened (SMO) gene in mouse and human lymphomas and, subsequently, to activate the GLI/Hh signalling [32].

In addition to basic research before, it has been noted that low expression of miR-200s clusters could correlate with adverse clinical outcome and serve as a prognostic biomarker for various cancer patients [15]. Although the potential prognostic value of $m i R-200 s$ clusters in several human cancers remains controversial, a recent meta-analysis demonstrated that lower tissue expression of miR-200s clusters' members were associated with poor OS and progression-free survival, whereas lower expression of circulating miR-200s clusters' members were correlated with favorable prognosis [15]. From our study, we showed the negative effect of low expression of miR-200s clusters on AML chemotherapy response and survival. Moreover, multivariate analysis showed that low expression of $m i R-429$ as an independent risk factor for $C R$, whereas low expression of $m i R-200 b$ as an independent prognostic biomarker for OS in AML. Due to some 

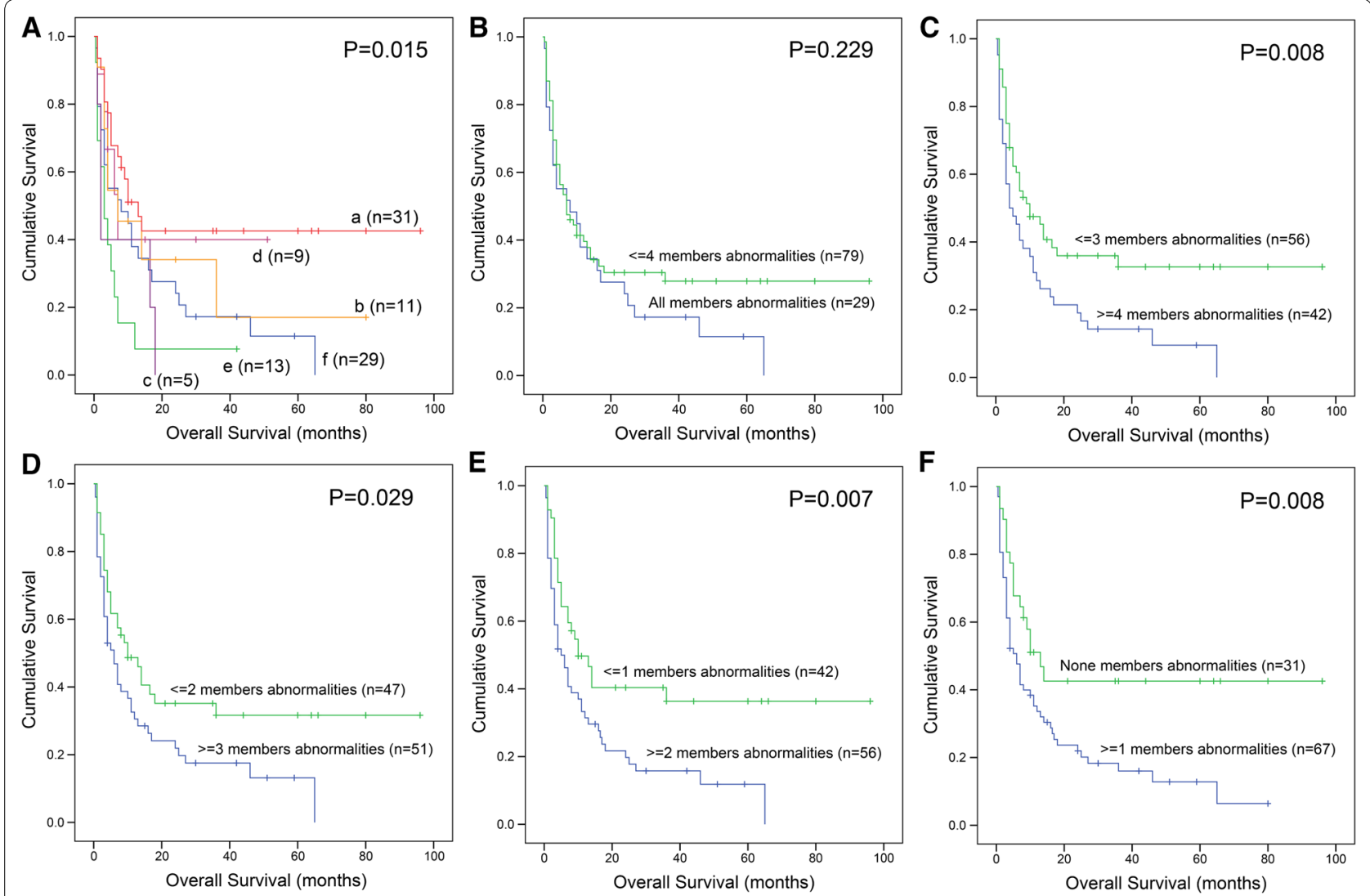

Fig. 3 Prognostic value of composite members of miR-200s clusters expression in AML. A Overall survival (OS) analyzed among AML patients with different numbers of abnormal miR-200s clusters expression; a: without miR-200s clusters abnormalities; b: with only one member of miR-200s clusters abnormalities; c: with two members of miR-200s clusters abnormalities; d: with three members of miR-200s clusters abnormalities; e: with four members of miR-200s clusters abnormalities; $f$ : with all members of miR-200s clusters abnormalities. B OS analyzed between two groups (all members of miR-200s clusters abnormalities vs. equal or less than four members of miR-200s clusters abnormalities). C OS analyzed between two groups (equal or more than four members of miR-200s clusters abnormalities vs. equal or less than three members of miR-200s clusters abnormalities). D OS analyzed between two groups (equal or more than three members of miR-200s clusters abnormalities vs. equal or less than two members of miR-200s clusters abnormalities). E OS analyzed between two groups (equal or more than two members of miR-200s clusters abnormalities vs. equal or less than one member of miR-200s clusters abnormalities). $\mathbf{F}$ OS analyzed between two groups (equal or more than one member of miR-200s clusters abnormalities vs. without any members of miR-200s clusters abnormalities)

limitations in this study (such as patients numbers, treatment regimens, and single center), prospective studies are needed to verify our results before $m i R$-200s clusters expression could be used routinely as a promising biomarker for risk stratification in AML.

\section{Conclusion}

Expression of $m i R-200 a / 200 b / 429$ cluster was frequently down-regulated in AML, and low expression of miR429 as an independent risk factor for CR, whereas low expression of $m i R-200 \mathrm{~b}$ as an independent prognostic biomarker for OS.

\section{Additional file}

Additional file 1: Table S1. The primer sequences for miR-200s clusters.

\section{Abbreviations}

AML: acute myeloid leukemia; $3^{\prime}$-UTR: $3^{\prime}$-untranslated region; APL: acute promyelocytic leukemia; BM: bone marrow; CR: complete remission; BMMNCs: BM mononuclear cells; FAB: French-American-British; WHO: World Health Organization; RT-qPCR: real-time quantitative PCR; OS: overall survival; WBC: white blood cell.

\section{Authors' contributions}

$J Q$ and $J L$ conceived and designed the experiments; $J Z$ and $L Z$ performed the experiments; JZ and TZ analyzed the data; $Y G, W Z$ and DW collected the clinical data; JM, XW and HG offered technique support; JZ wrote the paper. All authors read and approved the final manuscript.

\section{Author details}

${ }^{1}$ Department of Hematology, Affiliated People's Hospital of Jiangsu University, 8 Dianli Rd., Zhenjiang 212002, Jiangsu, People's Republic of China. ${ }^{2}$ The Key Lab of Precision Diagnosis and Treatment of Zhenjiang City, Zhenjiang, Jiangsu, People's Republic of China. ${ }^{3}$ Jingjiang College of Jiangsu University, Zhenjiang, Jiangsu, People's Republic of China. ${ }^{4}$ Department of Hematology, The Third People's Hospital of Kunshan City, Kunshan, Jiangsu, People's Republic of China. ${ }^{5}$ Laboratory Center, Affiliated People's Hospital of Jiangsu University, 8 Dianli Rd., Zhenjiang 212002, Jiangsu, People's Republic of China. 


\section{Acknowledgements \\ None.}

\section{Competing interests}

The authors declare that they have no competing interests.

\section{Availability of data and materials}

The datasets used and/or analysed during the current study are available from the corresponding author on reasonable request.

\section{Consent for publication}

Written informed consents were obtained from all enrolled individuals prior to their participation.

\section{Ethical approval and consent to participate}

The present study approved by the Ethics Committee and Institutional Review Board of the Affiliated People's Hospital of Jiangsu University.

\section{Funding}

This work was supported by National Natural Science foundation of China (81270630), Medical Innovation Team of Jiangsu Province (CXTDB2017002), 333 Project of Jiangsu Province (BRA2016131), Six Talent Peaks Project in Jiangsu Province (2015-WSN-115), Postgraduate Research \& Practice Innovation Program of Jiangsu Province (KYCX17_1821), Youth Medical Talents Project of "Ke Jiao Qiang Wei" project of Jiangsu province (QNRC2016450, QNRC2016449), China Postdoctoral Science Foundation funded project (2016M601748), Clinical Medical Science and Development Foundation of Jiangsu University (JLY20160011), Social Development Foundation of Kunshan (KS1624), Social Development Foundation of Zhenjiang (SH2016045, SH2016046, SH2017040), Key Medical Talent Program of Zhenjiang City.

\section{Publisher's Note}

Springer Nature remains neutral with regard to jurisdictional claims in published maps and institutional affiliations.

\section{Received: 23 March 2018 Accepted: 21 April 2018}

Published online: 21 May 2018

\section{References}

1. Döhner H, Weisdorf DJ, Bloomfield CD. Acute myeloid leukemia. N Engl J Med. 2015;373(12):1136-52.

2. Rowley JD. Chromosomal translocations: revisited yet again. Blood. 2008;112(6):2183-9.

3. Mrózek K, Marcucci G, Nicolet D, Maharry KS, Becker H, Whitman SP, Metzeler KH, Schwind S, Wu YZ, Kohlschmidt J, Pettenati MJ, Heerema NA, Block AW, Patil SR, Baer MR, Kolitz JE, Moore JO, Carroll AJ, Stone RM, Larson RA, Bloomfield CD. Prognostic significance of the European leukemia net standardized system for reporting cytogenetic and molecular alterations in adults with acute myeloid leukemia. J Clin Oncol. 2012;30(36):4515-23.

4. Marcucci G, Haferlach T, Döhner H. Molecular genetics of adult acute myeloid leukemia: prognostic and therapeutic implications. J Clin Oncol. 2011;29(5):475-86.

5. Patel JP, Levine RL. How do novel molecular genetic markers influence treatment decisions in acute myeloid leukemia? Hematol Am Soc Hematol Educ Program. 2012;2012:28-34.

6. Bartel DP. MicroRNAs: genomics, biogenesis, mechanism, and function. Cell. 2004;116(2):281-97.

7. Hwang HW, Mendell JT. MicroRNAs in cell proliferation, cell death, and tumorigenesis. Br J Cancer. 2006;94(6):776-80.

8. Trino S, Lamorte D, Caivano A, Laurenzana I, Tagliaferri D, Falco G, Del Vecchio L, Musto P, De Luca L. MicroRNAs as new biomarkers for diagnosis and prognosis, and as potential therapeutic targets in acute myeloid leukemia. Int J Mol Sci. 2018;19(2):460.

9. Ma QL, Wang JH, Yang M, Wang HP, Jin J. MiR-362-5p as a novel prognostic predictor of cytogenetically normal acute myeloid leukemia. J Transl Med. 2018;16(1):68.
10. Yan J, Wu G, Chen J, Xiong L, Chen G, Li P. Downregulated miR-217 expression predicts a poor outcome in acute myeloid leukemia. Cancer Biomark. 2018. https://doi.org/10.3233/CBM-170936.

11. Zhang TJ, Wu DH, Zhou JD, Li XX, Zhang W, Guo H, Ma JC, Deng ZQ, Lin J, Qian J. Overexpression of miR-216b: prognostic and predictive value in acute myeloid leukemia. J Cell Physiol. 2018;233(4):3274-81.

12. Bhayadia R, Krowiorz K, Haetscher N, Jammal R, Emmrich S, Obulkasim A, Fiedler J, Schwarzer A, Rouhi A, Heuser M, Wingert S, Bothur S, Döhner K, Mätzig T, Ng M, Reinhardt D, Döhner H, Zwaan CM, van den Heuvel Eibrink M, Heckl D, Fornerod M, Thum T, Humphries RK, Rieger MA, Kuchenbauer F, Klusmann JH. Endogenous tumor suppressor microRNA193b: therapeutic and prognostic value in acute myeloid leukemia. J Clin Oncol. 2018;36(10):1007-16.

13. Korpal M, Kang Y. The emerging role of miR-200 family of microRNAs in epithelial-mesenchymal transition and cancer metastasis. RNA Biol. 2008;5(3):115-9.

14. Feng $X$, Wang Z, Fillmore $R, X i$ Y. MiR-200, a new star miRNA in human cancer. Cancer Lett. 2014;344(2):166-73.

15. Lee JS, Ahn YH, Won HS, Sun S, Kim YH, Ko YH. Prognostic Role of the MicroRNA-200 family in various carcinomas: a systematic review and meta-analysis. Biomed Res Int. 2017;2017:1928021.

16. Bennett JM, Catovsky D, Daniel MT, Flandrin G, Galton DA, Gralnick HR, Sultan C. Proposed revised criteria for the classification of acute myeloid leukaemia. A report of the French-American-British Cooperative Group. Ann Intern Med. 1985;103(4):620-5

17. Arber DA, Orazi A, Hasserjian R, Thiele J, Borowitz MJ, Le Beau MM, Bloomfield CD, Cazzola M, Vardiman JW. The 2016 revision to the World Health Organization classification of myeloid neoplasms and acute leukemia. Blood. 2016;127(20):2391-405

18. Zhou JD, Zhang TJ, Li XX, Ma JC, Guo H, Wen XM, Zhang W, Yang L, Yan Y, Lin J, Qian J. Epigenetic dysregulation of ID4 predicts disease progression and treatment outcome in myeloid malignancies. J Cell Mol Med. 2017;21(8):1468-81.

19. Grimwade D, Hills RK, Moorman AV, Walker H, Chatters S, Goldstone AH, Wheatley K, Harrison CJ, Burnett AK, National Cancer Research Institute Adult Leukaemia Working Group. Refinement of cytogenetic classification in acute myeloid leukemia: determination of prognostic significance of rare recurring chromosomal abnormalities among 5876 younger adult patients treated in the United Kingdom Medical Research Council trials. Blood. 2010;116(3):354-65.

20. Cheng H, Huang C, Xu X, Hu X, Gong S, Tang G, Song X, Zhang W, Wang J. Chen L, Yang J. PIM-1 mRNA expression is a potential prognostic biomarker in acute myeloid leukemia. J Transl Med. 2017;15(1):179.

21. Lin J, Yao DM, Qian J, Chen Q, Qian W, Li Y, Yang J, Wang CZ, Chai HY, Qian Z, Xiao GF, Xu WR. Recurrent DNMT3A R882 mutations in Chinese patients with acute myeloid leukemia and myelodysplastic syndrome. PLOS ONE. 2011;6(10):e26906.

22. Lin J, Yao DM, Qian J, Chen Q, Qian W, Li Y, Yang J, Wang CZ, Chai HY, Qian Z, Xiao GF, Xu WR. IDH1 and IDH2 mutation analysis in Chinese patients with acute myeloid leukemia and myelodysplastic syndrome. Ann Hematol. 2012;91(4):519-25.

23. Yang X, Qian J, Sun A, Lin J, Xiao G, Yin J, Xiao G, Yin J, Chen S, Wu D. RAS mutation analysis in a large cohort of Chinese patients with acute myeloid leukemia. Clin Biochem. 2013;46(7-8):579-83.

24. Qian J, Yao DM, Lin J, Qian W, Wang CZ, Chai HY, Yang J, Li Y, Deng ZQ, Ma JC, Chen XX. U2AF1 mutations in Chinese patients with acute myeloid leukemia and myelodysplastic syndrome. PLoS ONE. 2012;7(9):e45760.

25. Lin J, Yang J, Wen XM, Yang L, Deng ZQ, Qian Z, Ma JC, Guo H, Zhang YY, Qian W, Qian J. Detection of SRSF2-P95 mutation by high-resolution melting curve analysis and its effect on prognosis in myelodysplastic syndrome. PLoS ONE. 2014:9(12):e115693.

26. Wen $X M$, Lin J, Yang J, Yao DM, Deng ZQ, Tang CY, Xiao GF, Yang L, Ma JC, Hu JB, Qian W, Qian J. Double CEBPA mutations are prognostically favorable in non-M3 acute myeloid leukemia patients with wild-type NPM1 and FLT3-ITD. Int J Clin Exp Pathol. 2014;7(10):6832-40.

27. Zhang TJ, Zhou JD, Zhang W, Lin J, Ma JC, Wen XM, Yuan Q, Li XX, Xu ZJ, Qian J. H19 overexpression promotes leukemogenesis and predicts 
unfavorable prognosis in acute myeloid leukemia. Clin Epigenetics. 2018;10:47.

28. Zhang TJ, Lin J, Zhou JD, Li XX, Zhang W, Guo H, Xu ZJ, Yan Y, Ma JC, Qian J. High bone marrow miR-19b level predicts poor prognosis and disease recurrence in de novo acute myeloid leukemia. Gene. 2018;640:79-85.

29. Li H, Mar BG, Zhang H, Puram RV, Vazquez F, Weir BA, Hahn WC, Ebert $B$, Pellman D. The EMT regulator ZEB2 is a novel dependency of human and murine acute myeloid leukemia. Blood. 2017;129(4):497-508.

30. Humphries B, Yang C. The microRNA-200 family: small molecules with novel roles in cancer development, progression and therapy. Oncotarget. 2015;6(9):6472-98.

31. Choi Y, Hur EH, Moon JH, Goo BK, Choi DR, Lee JH. Expression and prognostic significance of microRNAs in Korean patients with myelodysplastic syndrome. Korean J Intern Med. 2017. https://doi. org/10.3904/kjim.2016.239 (Epub ahead of print)

32. González-Gugel E, Villa-Morales M, Santos J, Bueno MJ, Malumbres M, Rodríguez-Pinilla SM, Piris MÁ, Fernández-Piqueras J. Down-regulation of specific miRNAs enhances the expression of the gene Smoothened and contributes to T-cell lymphoblastic lymphoma development. Carcinogenesis. 2013;34(4):902-8.
Ready to submit your research? Choose BMC and benefit from:

- fast, convenient online submission

- thorough peer review by experienced researchers in your field

- rapid publication on acceptance

- support for research data, including large and complex data types

- gold Open Access which fosters wider collaboration and increased citations

- maximum visibility for your research: over 100M website views per year

At BMC, research is always in progress.

Learn more biomedcentral.com/submissions 